\title{
Patient report
}

\section{Anke L.L. Lameris, Christel L.M. Geesing, Joost G.J. Hoenderop and Michiel F. Schreuder* Importance of dietary calcium and vitamin D in the treatment of hypercalcaemia in Williams-Beuren syndrome}

\begin{abstract}
Background: Williams-Beuren syndrome (WBS) is a rare genetic disorder caused by the deletion of 26-28 genes on chromosome 7. Fifteen percent of WBS patients present with hypercalcaemia during infancy, which is generally mild and resolves spontaneously before the age of 4 years. The mechanisms underlying the transient hypercalcaemia in WBS are poorly understood.

Case: We report a case of severe symptomatic hypercalcaemia in a patient with WBS, in which treatment with mild calcium restriction, hyperhydration and repeated bisphosphonate administration only resulted in short-lasting effects. Long-term lowering of serum calcium was only achieved after reducing calcium and vitamin D intake to the bare minimum.

Conclusions: This case illustrates the potential severity of hypercalcaemia in WBS, and demonstrates that both the cause as well as the solution of this problem may be found in the intestinal absorption of calcium. We hypothesise that the phenotypical resemblance between WBS and transient idiopathic infantile hypercalcaemia can be explained by similarities in the underlying genetic defect. Patients suffering from transient infantile hypercalcaemia were recently described to have mutations in CYP24A1, the key enzyme in 1,25-dihydroxyvitamin $\mathrm{D}_{3}$ degradation. In the light of this new development we discuss the role of one of the deleted genes in WBS, Williams syndrome transcription factor (WSTF), in the etiology of hypercalcaemia in WBS.
\end{abstract}

Keywords: CYP24A1; hypercalcaemia; Williams-Beuren syndrome (WBS); Williams syndrome transcription factor (WSTF).

\footnotetext{
*Corresponding author: Dr. Michiel F. Schreuder, Department of Pediatric Nephrology, Radboud University Medical Center, 804, PO Box 9101, 6500 HB Nijmegen, The Netherlands,

Phone: +31-24-361 4430, Fax: +31-24-361 6428,

E-mail: michiel.schreuder@radboudumc.nl
}

\begin{abstract}
Anke L.L. Lameris and Joost G.J. Hoenderop: Department of Physiology, Radboud University Medical Center, Nijmegen, The Netherlands

Christel L.M. Geesing: Department of Pediatrics, Bernhoven Hospital, Oss, The Netherlands
\end{abstract}

\section{Introduction}

Williams-Beuren syndrome (WBS, OMIM 194050) is a rare genetic disorder caused by a hemizygous deletion at 7q11.23 comprising approximately 26-28 genes (1). Patients with WBS are generally characterised by distinctive facial dysmorphisms, mental retardation and cardiovascular abnormalities. In addition, approximately $15 \%$ of WBS patients present with hypercalcaemia during infancy, which is generally mild in nature. It resolves spontaneously before the age of 4 years, but sometimes reoccurs briefly during puberty (1). The mechanisms explaining the transient hypercalcaemia are poorly understood. We report a case of severe symptomatic hypercalcaemia in a patient with WBS only resolving with a profound reduction in calcium and vitamin $\mathrm{D}$ intake, and present a potential mechanism for this metabolic abnormality.

\section{Case report}

An 18-month-old female patient was admitted to a local hospital to start tube feeding for treatment of failure to thrive due to feeding difficulties and persistent vomiting despite proton-pump inhibition. Three months earlier she was diagnosed with WBS with normal serum calcium levels $(2.40 \mathrm{mmol} / \mathrm{L})$, and was born with a low birth weight, a cardiac murmur without a structural anomaly, and a developmental delay.

During her admission, her condition deteriorated, and she developed hypercalcaemia (Table 1) and hypertension (up to $150 / 130 \mathrm{~mm} \mathrm{Hg}$ ) for which she was transferred to the paediatric intensive care unit at the Radboud 
Table 1 Laboratory parameters at diagnosis and during follow-up.

\begin{tabular}{|c|c|c|c|c|c|}
\hline Parameter & Normal range & $\begin{array}{l}\text { Hospital admission } \\
\text { for hypercalcaemia }\end{array}$ & $\begin{array}{r}3 \text { months after } \\
\text { admission }\end{array}$ & $\begin{array}{r}6 \text { months after } \\
\text { admission }\end{array}$ & Latest follow-up \\
\hline Age & & 1 year, 6 months & 1 year, 9 months & 2 years, 1 month & 4 years, 6 months \\
\hline Total calcium, mmol/L & $2.20-2.70$ & 3.51 & 2.61 & 2.76 & 2.73 \\
\hline Calcium ion, $\mathrm{mmol} / \mathrm{L}$ & $1.10-1.30$ & ND & 1.33 & 1.37 & 1.26 \\
\hline Albumin, $\mathrm{g} / \mathrm{L}$ & $35-50$ & 45.0 & 44 & 52 & ND \\
\hline Phosphate, $\mathrm{mmol} / \mathrm{L}$ & $1.5-2.2$ & 1.5 & 1.72 & 1.54 & 1.26 \\
\hline $\mathrm{eGFR}, \mathrm{mL} / \mathrm{min} / 1.73 \mathrm{~m}^{2}$ & $90-120$ & 41 & 129 & 114 & ND \\
\hline 25-(OH)-vitamin D, nmol/L & $18-113$ & 56.7 & 46 & 38 & ND \\
\hline 1,25-(OH)-vitamin D, pmol/L & $47-130$ & 18.7 & ND & 250 & ND \\
\hline PTH, pmol/L & $1.3-6.8$ & 0.4 & 9.9 & 6.2 & 2.7 \\
\hline $\begin{array}{l}\text { Urine calcium-creatinine ratio, } \\
\mathrm{mmol} / \mathrm{mmol}\end{array}$ & $<1.5$ & 1.15 & $<0.3$ & $<0.3$ & 0.55 \\
\hline
\end{tabular}

ND, not determined; eGFR, estimated glomerular filtration rate based on the Schwartz equation; PTH, parathyroid hormone.

Radboud University Medical Center. Her blood pressure was controlled with an intravenous calcium-antagonist, which was successfully switched to oral anti-hypertensive agents (amlodipine and labetalol). Extensive evaluation, excluding renal angiography, did not show an explanation for the hypertension, other than the hypercalcaemia and the underlying WBS.

The hypercalcaemia was treated with hyperhydration, furosemide and a reduced calcium intake. Laboratory tests ruled out other causes than the WBS for the hypercalcaemia (Table 1). To prevent nephrocalcinosis due to the subsequent hypercalciuria (urine calcium-creatinine ratio up to $3.0 \mathrm{mmol} / \mathrm{mmol}$ ) potassium citrate was administered.

As this treatment only led to a small reduction in serum calcium (Figure 1), a bisphosphonate was administered in a low dose to prevent hypocalcaemia. This resulted in only a short and mild reduction in the serum calcium; so repeated and increased doses of pamidronate were administered, all with only short-lasting effects (Figure 1). With every return of hypercalcaemia, the patient also showed an increase in blood pressure and vomiting. Therefore, calcium and vitamin D intake (drip-feed containing $588 \mathrm{mg} /$ day and $150 \mathrm{IE} /$ day, respectively) was reduced to the bare minimum ( $68 \mathrm{mg} /$ day and $0 \mathrm{IE} /$ day, respectively) while maintaining adequate nutritional intake. With this treatment, serum calcium was maintained at high-normal but stable levels that were clinically well accepted. Phosphate supplementation was initiated to treat her accompanying hypophosphataemia.

During follow-up, we found to our surprise a low renal calcium excretion and high parathyroid hormone (PTH) level during the calcium restriction. We interpreted this to be indicative of a more calcium-deficient state, despite the high-normal serum calcium, for which the calcium intake was gradually increased. No nephrocalcinosis is present at the latest ultrasound, and no hypercalciuria occurred during the gradual increase in calcium intake.

\section{Discussion}

In the past, various studies have been performed to try and elucidate the mechanism underlying hypercalcaemia in WBS. We hypothesise that increased intestinal absorption of calcium is the main factor in the pathogenesis of hypercalcaemia in these patients, as has been described in the past (2), potentially as a result of reduced breakdown of vitamin D. Most studies aimed to find changes in vitamin D metabolism, with various degrees of success. Some groups find abnormalities in all patients suffering from WBS, whereas others only describe changes in vitamin D metabolism in WBS patients with hypercalcaemia, and some groups find no changes at all (3).

Bisphosphonate therapy (pamidronate) is recommended as an additional therapy when conventional measures (intravenous fluids, furosemide and dietary calcium restriction) fail to normalise serum calcium. However, the course of the hypercalcaemia in our patient seems to suggest no sustained effect of bisphosphonate treatment and suggests an intestinal cause of hypercalcaemia that can only be treated by (extensive) calcium and vitamin D restriction. It is to be noted that our patient did not show extensive hypercalciuria at presentation (Table 1), which would be expected in hypercalcaemia. However, renal function was reduced at that time with an 


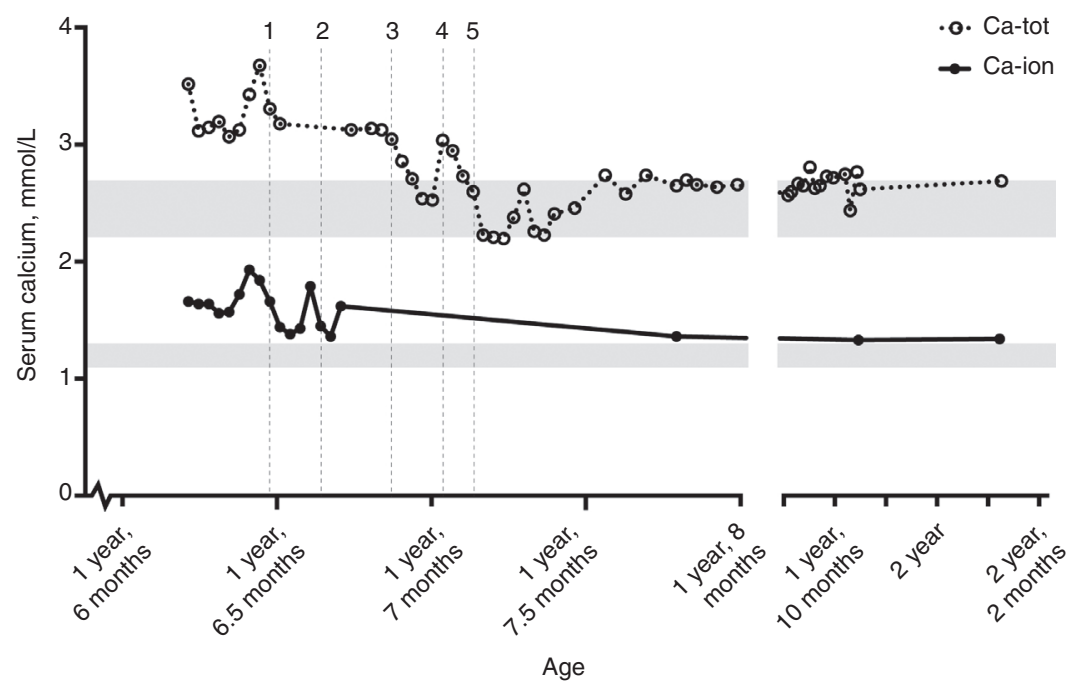

Figure 1 Time course of total calcium and ionised calcium levels in serum during treatment and follow-up. Grey areas indicate the normal range total serum calcium ( $2.20-2.70 \mathrm{mmol} / \mathrm{L})$ and ionised calcium (1.10-1.30 mmol/L). The arrows indicate interventions. 1-4: Intravenous administration of pamidronate, $0.25,0.50,1.0$, and $1.0 \mathrm{mg}$ pamidronate/ $\mathrm{kg}$ body weight, respectively. 5: Diet changed from drip-feed containing $588 \mathrm{mg} \mathrm{Ca} /$ day and 150 IE 1,25-dihydroxyvitamin $\mathrm{D}_{3} /$ day to a drip-feed with $68 \mathrm{mg}$ Ca/day and 0 IE 1,25-dihydroxyvitamin $D_{3}$ /day.

estimated glomerular filtration rate of $41 \mathrm{~mL} / \mathrm{min} / 1.73 \mathrm{~m}^{2}$ and hypercalciuria did present after optimising renal function (urine calcium-creatinine ratio up to $3.0 \mathrm{mmol} / \mathrm{mmol}$ ). The reduced renal function may explain the temporary absence of hypercalciuria, and may have contributed to the observed hypercalcaemia. The persisting hypercalciuria as well as hypercalcaemia that were observed when renal function was restored indicate that the hypercalcaemia in our patient is not solely explained by inappropriate renal calcium handling due to renal failure. Some of the features of our patient are not easily explained and may indicate an additional factor involved in the aetiology of hypercalcaemia in this particular patient. The high PTH levels observed after 3-6 months, when serum calcium levels are in the high-normal range could also indicate an altered set point of serum calcium, which is commonly associated with inactivating mutations in the calciumsensing receptor (CaSR).

Hypertension is, in addition to the hypercalcaemia, a well-known feature in up to $50 \%$ of WBS patients and may be explained by elastin deficiency, a hallmark feature of WBS (1). Alternatively, renal artery stenosis is described in some patients with WBS, which we did not fully exclude in our patient yet. As blood pressure was well controlled with the current medical therapy, renal ultrasound showed identical-sized and normal-structured kidneys, and renography showed a normal split renal function; no renal angiography was performed.

Our case of severe and sustained hypercalcaemia highlights that the underlying mechanism in WBS can be found, at least in part, in the uptake of calcium, as the long-term lowering of serum calcium was only obtained by a subtotal reduction in the calcium and vitamin $\mathrm{D}$ intake. Hypercalcaemia in WBS patients and patients suffering from IIH without WBS both have been shown to result from intestinal hyperabsorption of calcium, the contribution of potential changes in renal calcium handling to the observed hypercalcaemia remains to be determined (2).

Similar to our WBS patient, IIH patients were shown to benefit from dietary calcium and vitamin $\mathrm{D}$ restriction (4). Patients should, however, be carefully monitored as long-term restriction of dietary calcium and vitamin $\mathrm{D}$ may induce rickets in these young patients $(4,5)$.

Based on these phenotypical similarities between patients with WBS and IIH, we previously suggested there might be a common underlying genetic defect (6). We hypothesised that claudin 3 and claudin 4, both involved in paracellular calcium absorption and located within the hemizygously deleted WBS region, might be responsible for the hypercalcaemia state of these patients. Unfortunately, we were not able to confirm this hypothesis in patients suffering from isolated infantile hypercalcaemia (6). Letavernier et al. suggested that hypercalcaemia in WBS patients is the result of TRPC3 overexpression due to haploinsufficiency of the transcriptions factor TFII-I, which is located in the genomic region that is deleted in WBS patients (7). Evidence that TRPC3 is involved in transepithelial calcium transport in the intestine, however, is lacking. Despite the earlier negative findings, our 
hypothesis remains valid. The similarities with regard to the transient hypercalcaemic phenotype of IIH and WBS patients suggest a common underlying genetic defect. Interestingly, the genetic defect causing IIH has recently been elucidated, shedding more light on the molecular mechanism underlying hypercalcaemia in these patients. Two independent studies have found that homozygous or compound heterozygous mutations and deletions in the CYP24A1 gene are responsible for the phenotype of patients suffering from IIH. CYP24A1 encodes 25-dihydroxyvitamin D 24-hydroxylase, which is the key enzyme in 1,25-dihydroxyvitamin $\mathrm{D}_{3}$ degradation $(8,9)$. Patients display hypercalcaemia, hypercalciuria, and suppressed PTH levels, whereas levels of 25-hydroxyvitamin $\mathrm{D}_{3}$ and 1,25-dihydroxyvitamin $\mathrm{D}_{3}$ were within normal range. A third study describes patients with a heterozygous mutation in CYP24A1 that has a less severe clinical phenotype compared to the compound heterozygous patients, indicative of a haploinsufficiency or a dominant negative effect (10). This study also shows that patients with CYP24A1 mutations have reduced levels of circulating 24,25-dihydroxyvitamin $\mathrm{D}_{3}$ in line with a non-functional Cyp24a1 enzyme. Together, these studies indicate that in IIH patients, intestinal hyperabsorption of calcium is the result of altered vitamin D catabolism.

Interestingly, a gene named WSTF is located within the WBS deletion region. WSTF is a subunit of the ATPdependent chromatin remodeling complex WINAC, whose activity facilitates ligand-dependent activation of CYP24A1 and repression of 25-hydroxyvitamin $\mathrm{D}_{3}$-1-alpha hydroxylase (CYP27B1) by the vitamin D receptor (11).

It has been previously hypothesised that hypercalcaemia in WBS is the result of loss of WINAC function, leading to reduced transcription of CYP24A1 and increased transcription of CYP27B1, theoretically resulting in increased 1,25-dihydroxyvitamin $\mathrm{D}_{3}$ (12). Lack of a clear increase in 1,25-dihydroxyvitamin $\mathrm{D}_{3}$ levels in WBS patients seems to contradict this hypothesis. However, the phenotype of patients with CYP24A1 mutations clearly demonstrates that a lack of 25-dihydroxyvitamin D 24-hydroxylase may indeed lead to a hypercalcaemic phenotype without a clear increase in circulating 1,25-dihydroxyvitamin $\mathrm{D}_{3}$ levels; only the patients receiving large doses of vitamin D prophylaxis display elevated levels of circulating 1,25-dihydroxyvitamin $\mathrm{D}_{3}$ (9). As our patient was admitted for failure to thrive, it is safe to assume that her vitamin D intake was low. Similar observations have been made in Cyp24a1 knockout mice, which have normal 1,25-dihydroxyvitamin $\mathrm{D}_{3}$ levels at baseline, but show an impaired clearance of administered 1,25-dihydroxyvitamin $\mathrm{D}_{3}$ (13). The exact explanation for this finding remains to be determined, but it strengthens the hypothesis that the hemizygous deletion of WSTF could be responsible for the transient hypercalcaemia in WBS, despite a lack of clear alterations in 1,25-dihydroxyvitamin $\mathrm{D}_{3}$. These data are in line with our hypothesis that deletion of WSTF in WBS patients leads to a reduced CYP24A1 activity, therefore, a reduced catabolism of 1,25-dihydroxyvitamin $\mathrm{D}_{3}$, which results in increased intestinal calcium absorption with subsequent hypercalcaemia.

In conclusion, our patient illustrates the potential severity of hypercalcaemia in WBS, and demonstrates that both the cause and the solution of this problem may be found in the intestinal absorption of calcium. Key to treatment of severe hypercalcaemia in WBS is subtotal restriction of calcium and vitamin D intake. Follow-up should include careful monitoring of serum and urinary calcium levels, to navigate between hypercalcaemia and calcium depletion.

\section{Support and Financial Disclosure}

None of the authors received support for the current manuscript. None of the authors has a potential conflict of interest to declare.

Received June 4, 2013; accepted January 14, 2014; previously published online February 27, 2014

\section{References}

1. Pober BR. Williams-Beuren syndrome. N Engl J Med 2010;362:239-52.

2. Barr DG, Forfar JO. Oral calcium-loading test in infancy, with particular reference to idiopathic hypercalcaemia. Br Med J 1969;1:477-80.

3. Kruse K, Pankau R, Gosch A, Wohlfahrt K. Calcium metabolism in Williams-Beuren syndrome. J Pediatr 1992;121:902-7.
4. Martin ND, Snodgrass GJ, Cohen RD. Idiopathic infantile hypercalcaemia-a continuing enigma. Arch Dis Child 1984;59:605-13.

5. Gut J, Kutilek S. Idiopathic infantile hypercalcaemia in 5-month-old girl. Prague Med Rep 2011;112:124-31.

6. Lameris AL, Huybers S, Burke JR, Monnens LA, Bindels RJ, Hoenderop JG. Involvement of claudin 3 and claudin 4 in 
idiopathic infantile hypercalcaemia: a novel hypothesis? Nephrol Dial Transplant 2010;25:3504-9.

7. Letavernier E, Rodenas A, Guerrot D, Haymann JP. WilliamsBeuren syndrome hypercalcemia: is TRPC3 a novel mediator in calcium homeostasis? Pediatrics 2012;129:e1626-30.

8. Dauber A, Nguyen TT, Sochett E, Cole DE, Horst R, et al. Genetic defect in CYP24A1, the vitamin D 24-hydroxylase gene, in a patient with severe infantile hypercalcemia. J Clin Endocrinol Metab 2012;97:E268-74.

9. Schlingmann KP, Kaufmann M, Weber S, Irwin A, Goos C, et al. Mutations in CYP24A1 and idiopathic infantile hypercalcemia. N Engl J Med 2011;365:410-21.

10. Tebben PJ, Milliner DS, Horst RL, Harris PC, Singh RJ, et al. Hypercalcemia, hypercalciuria, and elevated calcitriol concentrations with autosomal dominant transmission due to CYP24A1 mutations: effects of ketoconazole therapy. J Clin Endocrinol Metab 2012;97:E423-7.

11. Barnett C, Krebs JE. WSTF does it all: a multifunctional protein in transcription, repair, and replication. Biochem Cell Biol 2011;89:12-23.

12. Yoshimura K, Kitagawa H, Fujiki R, Tanabe M, Takezawa S, et al. Distinct function of 2 chromatin remodeling complexes that share a common subunit, Williams syndrome transcription factor (WSTF). Proc Natl Acad Sci USA 2009;106:9280-5.

13. St-Arnaud R, Arabian A, Travers R, Barletta F, Raval-Pandya M, et al. Deficient mineralization of intramembranous bone in vitamin D-24-hydroxylase-ablated mice is due to elevated 1,25-dihydroxyvitamin $D$ and not to the absence of 24,25-dihydroxyvitamin D. Endocrinology 2000;141:2658-66. 